\title{
Lattice Boltzmann simulation of liquid falling on horizontal rectangular pillar arrays
}

\author{
Makoto Sugimoto, Tatsuya Miyazaki, Zelin Li, Masayuki Kaneda*, and Kazuhiko Suga \\ Department of Mechanical Engineering, Osaka Prefecture University, 1-1 Gakuen-cho, Naka-ku, Sakai, 599-8531, Japan
}

\begin{abstract}
Stator coils of automobiles in operation generate heat and are cooled by a coolant poured from above. Since the behavior characteristic of the coolant poured on the coils is not clarified yet due to its complexity, the three-dimensional two-phase flow simulation is conducted. In this study, as a steppingstone to the simulation of the liquid falling on the actual coils, the coils are modelled with horizontal rectangular pillar arrays whose governing parameters can be easily changed. The two-phase flows are simulated using the lattice Boltzmann method and the phase-field model, and the effects of the governing parameters, such as the physical properties of the cooling liquid, the wettability, and the gap between the pillars, on the wetting area are investigated. The results show that the oil tends to spread across the pillars because of its high viscosity. Moreover, the liquid spreads quickly when the contact angle is small. In the case that the pillars are stacked, the wetting area of the inner pillars is larger than that of the exposed pillars.
\end{abstract}

\section{Introduction}

The electrification of automobiles is now increasing for $\mathrm{CO}_{2}$ reduction. The powertrains with high efficiency and high power density are necessary for further electrifications. One of the motor heat sources is the Joule heat of the coil. To increase the power density, cooling technology is important. Various cooling systems exist, air cooling, water cooling, and oil cooling. Oil is the insulating liquid and it can cool the coil directly. The oil cooling system enables efficient heat exchange because of the high heat transfer coefficient between the coils and the oil. To improve the cooling performance of the oil cooling system, it is necessary to maximize the contact area of the coils and the oil and to exhaust the warmed oil efficiently.

Since the actual coils inside the motor have a complex structure, the liquid flow mechanisms around the coils are not clarified yet. Moreover, the cooling performance is affected by the various governing parameters such as the wettability, the gap among the coils, and the physical properties of the cooling liquid. Therefore a numerical approach is required.

In this study, as a steppingstone to the simulation of the liquid falling on the actual coils, the coils are modeled with horizontal rectangular pillar arrays whose governing parameters can be easily changed, and the two-phase flows are simulated using a combination of the lattice Boltzmann method (LBM) and the phasefield model (PFM) $[1,2]$. Furthermore, the effects of the governing parameters on the wetting area are investigated.

\section{Governing equations}

The PFM is based on the free energy theory [3] of nonequilibrium system. In the PFM, the liquid and gas phases are distinguished using the order parameter $\phi$, which is defined as a local liquid volume fraction. Therefore, $\phi$ takes values of 0 and 1 for the gas and liquid phases, respectively. The region where $0<\phi<$ 1 is a diffuse interface with finite thickness $W$. The time evolution of the order parameter is expressed by the conservative Allen-Cahn equation, as follows $[1,2]$ :

$$
\frac{\partial \phi}{\partial t}+\boldsymbol{\nabla} \cdot(\phi \mathbf{u})=\boldsymbol{\nabla} \cdot\left[M_{\phi}(\boldsymbol{\nabla} \phi-\lambda \mathbf{n})\right]
$$

where $M_{\phi}$ is the mobility, $\lambda=4 \phi(1-\phi) / W$, and $\mathbf{n}=$ $\nabla \phi /|\nabla \phi|$ is the unit vector oriented normal to the liquidgas interface.

The time evolution of the velocity $\mathbf{u}$ and the pressure $p$ is expressed by the Navier-Stokes equation, as follows:

$\frac{\partial(\rho \mathbf{u})}{\partial t}+\boldsymbol{\nabla} \cdot(\rho \mathbf{u u})=-\nabla p+\boldsymbol{\nabla} \cdot\left[\mu\left(\boldsymbol{\nabla u}+\boldsymbol{\nabla} \mathbf{u}^{\mathrm{T}}\right)\right]+\mathbf{F}$,

where $\mathbf{F}$ denotes the total force term, which can be divided into the surface tension force $\mathbf{F}_{\mathrm{S}}$ and the gravitational force $\mathbf{F}_{\mathrm{g}}=\rho \mathbf{G}$, as follows:

$$
\mathbf{F}=\mathbf{F}_{\mathrm{s}}+\mathbf{F}_{\mathrm{g}} \text {. }
$$

In this study, the following potential form is used for the surface tension force:

$$
\mathbf{F}_{\mathrm{s}}=\mu_{\phi} \boldsymbol{\nabla} \phi
$$

where $\mu_{\phi}$ is the chemical potential, which is defined as follows:

$$
\mu_{\phi}=2 \beta \phi(1-\phi)(1-2 \phi)-\kappa \nabla^{2} \phi,
$$

*Corresponding author: mkaneda@me.osakafu-u.ac.jp 
where $\beta$ and $\kappa$ are the physical parameters below, which are dependent on both the interfacial thickness $W$ and the surface tension $\sigma$ :

$$
\beta=\frac{12 \sigma}{W}, \quad \kappa=\frac{3}{2} \sigma W .
$$

\section{Lattice Boltzmann method (LBM)}

The conservative Allen-Cahn equation and the NavierStokes equation are converted into the following lattice Boltzmann equations, respectively:

$$
\begin{aligned}
f_{i}\left(\mathbf{x}+\mathbf{e}_{i} \delta_{t}, t+\right. & \left.\delta_{t}\right)-f_{i}(\mathbf{x}, t) \\
& =\Omega_{i}^{f}(\mathbf{x}, t)+\delta_{t} R_{i}^{f}(\mathbf{x}, t), \\
g_{i}\left(\mathbf{x}+\mathbf{e}_{i} \delta_{t}, t+\right. & \left.\delta_{t}\right)-g_{i}(\mathbf{x}, t) \\
& =\Omega_{i}^{g}(\mathbf{x}, t)+\delta_{t} R_{i}^{g}(\mathbf{x}, t),
\end{aligned}
$$

where $f_{i}$ and $g_{i}$ are the distribution functions of the order parameter and the velocity, respectively. $\Omega_{i}^{f}$ and $\Omega_{i}^{g}$ are the collision operators. $R_{i}^{f}$ and $R_{i}^{g}$ are the source term and the force term, respectively. The subscript $i$ denotes the discrete direction, $\mathbf{e}_{i}$ is the discrete velocity, and $\delta_{t}$ is the time step.

In this study, the single-relaxation-time (SRT) model [4] and the weighted multiple-relaxation-time (WMRT) model [5] are used for the collision operators of the distribution functions $f_{i}$ and $g_{i}$, respectively. The collision operators are given as follows:

$$
\begin{gathered}
\mathbf{\Omega}^{f}=-\frac{1}{\tau_{f}}\left(\mathbf{f}-\mathbf{f}^{\mathrm{eq}}\right), \\
\mathbf{\Omega}^{g}=-\mathbf{M}^{-1} \mathbf{S}^{g} \mathbf{M}\left(\mathbf{g}-\mathbf{g}^{\mathrm{eq}}\right),
\end{gathered}
$$

where the superscript eq denotes the local equilibrium distribution function and $\mathbf{M}$ denotes the transformation matrix of the WMRT model. $\mathbf{S}^{g}$ is the diagonal collision matrix and is given as follows:

$$
\mathbf{S}^{g}=\operatorname{diag}(\underbrace{1, \ldots, 1}_{d+1}, \underbrace{s_{g}, \ldots, s_{g}}_{\frac{(d+2)(d-1)}{2}}, \underbrace{1, \ldots, 1}_{q-\frac{d(d+3)}{2}}),
$$

where $s_{g}=1 / \tau_{g} . d$ and $q$ denote the dimension of the discrete velocity model and the number of the discrete directions, respectively. The relaxation times $\tau_{f}$ and $\tau_{g}$ have the following relationships with the mobility and the kinematic viscosity, respectively:

$$
\begin{gathered}
M_{\phi}=c_{\mathrm{s}}^{2}\left(\tau_{f}-\frac{1}{2}\right) \delta_{t}, \\
\nu=c_{\mathrm{s}}^{2}\left(\tau_{g}-\frac{1}{2}\right) \delta_{t},
\end{gathered}
$$

where $c_{\mathrm{s}}$ denotes the speed of sound.

The local equilibrium distribution function $f_{i}^{\text {eq }}$ is obtained as follows using the first-order term of the Maxwell distribution:

$$
f_{i}^{\mathrm{eq}}=w_{i} \phi\left(1+\frac{\mathbf{e}_{i} \cdot \mathbf{u}}{c_{\mathrm{s}}^{2}}\right) .
$$

The local equilibrium distribution function $g_{i}^{\text {eq }}$ is written as $[1,2,6]$

$$
g_{i}^{\mathrm{eq}}= \begin{cases}\frac{p}{c_{\mathrm{s}}^{2}}\left(w_{i}-1\right)+\rho s_{i}(\mathbf{u}), & (i=0), \\ \frac{p}{c_{\mathrm{s}}^{2}} w_{i}+\rho s_{i}(\mathbf{u}), & (i \neq 0),\end{cases}
$$

where $w_{i}$ is the weight coefficient and $i=0$ is the discrete direction in which $\mathbf{e}_{i}=\mathbf{0} . s_{i}$ is the following function:

$$
s_{i}(\mathbf{u})=w_{i}\left[\frac{\mathbf{e}_{i} \cdot \mathbf{u}}{c_{\mathrm{s}}^{2}}+\frac{\left(\mathbf{e}_{i} \cdot \mathbf{u}\right)^{2}}{2 c_{\mathrm{s}}^{4}}-\frac{\mathbf{u} \cdot \mathbf{u}}{2 c_{\mathrm{s}}^{2}}\right] .
$$

The source term $R_{i}^{f}$ and the force term $R_{i}^{g}$ are requied to recover the governing equations from the LBMs and are expressed as follows [7]:

$$
\begin{gathered}
\mathbf{R}^{f}=\left(1-\frac{1}{2 \tau_{f}}\right) \overline{\mathbf{R}}^{f}, \\
\mathbf{R}^{g}=\mathbf{M}^{-1}\left(\mathbf{I}-\frac{\mathbf{S}^{g}}{2}\right) \mathbf{M} \overline{\mathbf{R}}^{g},
\end{gathered}
$$

where

$$
\begin{gathered}
\bar{R}_{i}^{f}=w_{i} \mathbf{e}_{i} \cdot\left[\frac{\partial_{t}(\phi \mathbf{u})}{c_{\mathrm{s}}^{2}}+\lambda \mathbf{n}\right], \\
\bar{R}_{i}^{g}=w_{i} \frac{\mathbf{e}_{i} \cdot \mathbf{F}+\left(\mathbf{e}_{i} \cdot \mathbf{u}\right)\left(\mathbf{e}_{i} \cdot \boldsymbol{\nabla} \rho\right)}{c_{\mathrm{s}}^{2}} .
\end{gathered}
$$

In this study, the time derivative term in Eq. (19) is computed using the following explicit Euler scheme:

$$
\partial_{t}(\phi \mathbf{u})=\frac{\phi(t) \mathbf{u}(t)-\phi\left(t-\delta_{t}\right) \mathbf{u}\left(t-\delta_{t}\right)}{\delta_{t}}
$$

The macroscopic variables, comprising the order parameter, the velocity, and the pressure, are computed using the zeroth or first moments of the distribution functions as follows:

$$
\begin{gathered}
\phi=\sum_{i} f_{i}, \\
\mathbf{u}=\frac{1}{\rho}\left(\sum_{i} \mathbf{e}_{i} g_{i}+\frac{\delta_{t}}{2} \mathbf{F}\right), \\
p=\frac{c_{\mathrm{s}}^{2}}{1-w_{0}}\left[\sum_{i \neq 0} g_{i}+\rho s_{0}(\mathbf{u})+\frac{\delta_{t}}{2} \mathbf{u} \cdot \boldsymbol{\nabla} \rho\right] .
\end{gathered}
$$

Furthermore, the density and the kinematic viscosity are interpolated using the order parameter as follows:

$$
\begin{gathered}
\rho=\phi \rho_{\mathrm{l}}+(1-\phi) \rho_{\mathrm{g}}, \\
v=\phi v_{\mathrm{l}}+(1-\phi) v_{\mathrm{g}},
\end{gathered}
$$

where the subscript 1 and $g$ denote the liquid and gas phases, respectively.

In this study, the D3Q27 discrete velocity model [8] is used for the distribution functions.

\section{Simplified model of coils}

In this study, the actual coils inside the motor are modeled with the horizontal rectangular pillar arrays shown in Fig. 1. The gaps between the pillars are set to $1 \mathrm{~mm}$. The inlet of the liquid is located at the center of the $+z$ boundary. The inlet is a circle with a diameter of about $3 \mathrm{~mm}$, and the flow rate is set to $0.5 \mathrm{~L} / \mathrm{min}$.

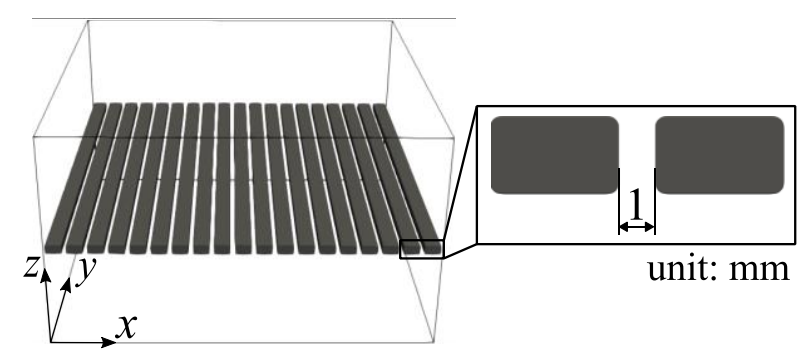

Fig. 1. Schematic drawing of the single-layered simplified model. 


\section{Results and discussion}

\subsection{Single-layered model}

The firstly-investigated schematic model of singlelayered model is shown in Fig. 1. The effects of the physical properties of the falling liquid, the wettability, and the gaps between the pillars on the wetting area on the pillars are discussed here.

\subsubsection{Effect of the falling liquid}

The cases of different liquid are simulated. The presumed liquids are the water and the oil. In this study, the gas phase is set to the air. The computational parameters are shown in Table 1. $W e=\rho_{\mathrm{l}} D U^{2} / \sigma$ is the Weber number, $O h=\mu_{\mathrm{l}} / \sqrt{\rho_{\mathrm{l}} \sigma D}$ is the Ohnesorge number, and $B o=\left(\rho_{\mathrm{l}}-\rho_{\mathrm{g}}\right)|\mathbf{G}| D^{2} / \sigma$ is the Bond number, where $D$ and $U$ denote the inlet diameter and the inlet velocity, respectively. In these simulations, the contact angle is set to $\theta=90^{\circ}$. Figure 2 shows the snapshots of the water and oil falling on the simplified model. It is confirmed that the water spreads along the center pillar instead of flowing across the gaps. On the other hand, the oil steps over the gaps and spreads while retaining the elliptical shape. These are because the high viscosity of the oil suppresses the spreading along the pillar, and the dammed oil flows across the pillars. This result indicates that, in terms of wetting, the oil is superior to the water for cooling the coils.

Table 1. Computational parameters.

\begin{tabular}{cccccc}
\hline Liquid & $\rho_{\mathrm{l}} / \rho_{\mathrm{g}}$ & $\mu_{\mathrm{l}} / \mu_{\mathrm{g}}$ & $W e$ & $O h$ & $B o$ \\
\hline Water & 828 & 55.5 & 70.4 & $2.22 \times 10^{-3}$ & 1.05 \\
Oil & 705 & 2360 & 121 & $1.47 \times 10^{-1}$ & 1.81 \\
\hline
\end{tabular}

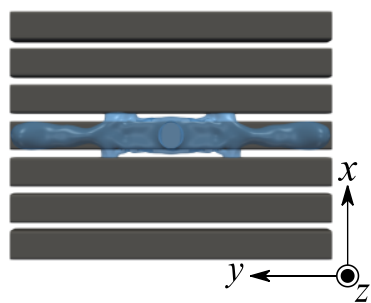

(a) Water.

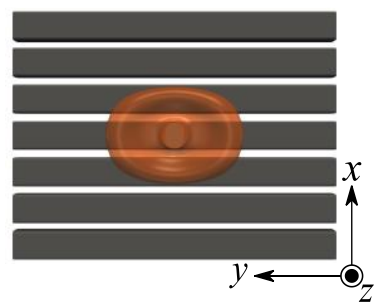

(b) Oil.
Fig. 2. Snapshots of the water and oil falling on the simplified model.

\subsubsection{Effect of the wettability}

In this section, the simulations are performed by changing the contact angle to $\theta=30^{\circ}, 60^{\circ}$ from the simulation of the oil in the previous section. Moreover, for validation, the numerical results are compared with the experimental one. Figures 3 and 4 show the numerical and experimental results of the oil spreading on the simplified model, respectively. In the experiment, the contact angle is less than $30^{\circ}$. These figures show that the wetting area of the numerical result with $\theta=$ $30^{\circ}$ has an elliptical shape, and qualitatively agrees with the experimental one. Figure 5 shows the transition of the wetting area for each contact angles, where $S_{\text {wet }}$ and

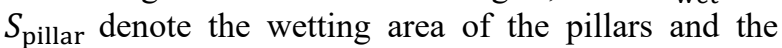
cross-sectional area of the pillar. From Fig. 5, it is confirmed that the oil falling on the lyophilic pillars spreads across the pillars at an early stage.

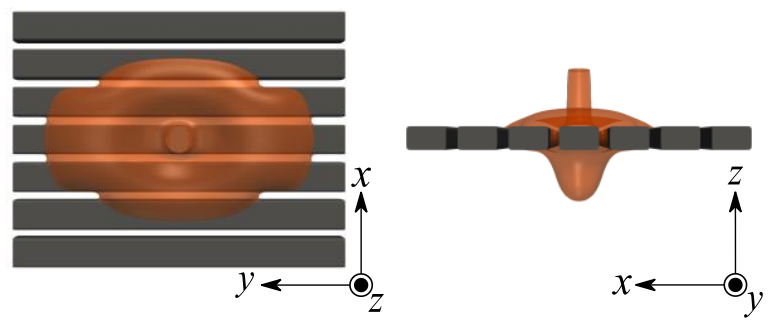

(a) $\theta=30^{\circ}$.

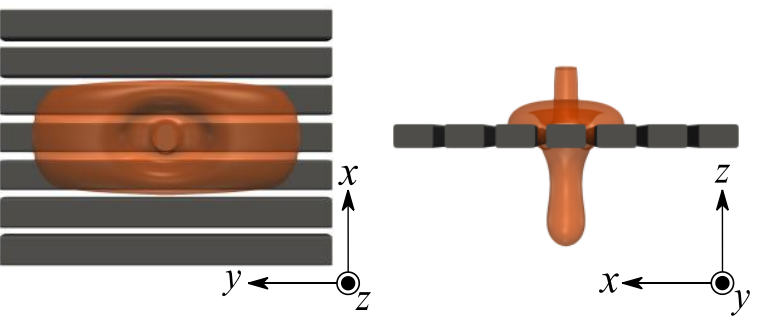

(b) $\theta=60^{\circ}$.
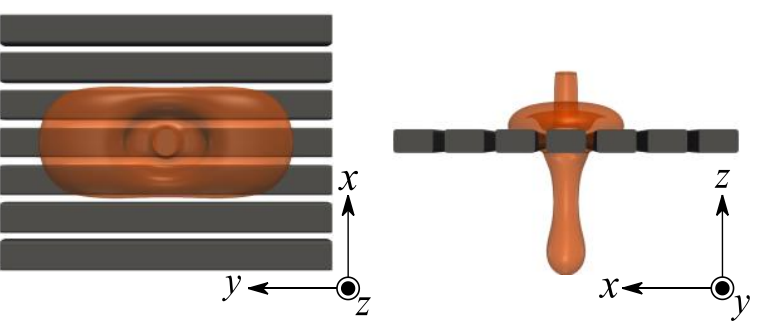

(c) $\theta=90^{\circ}$.

Fig. 3. Numerical results of the oil spreading on the simplified model.

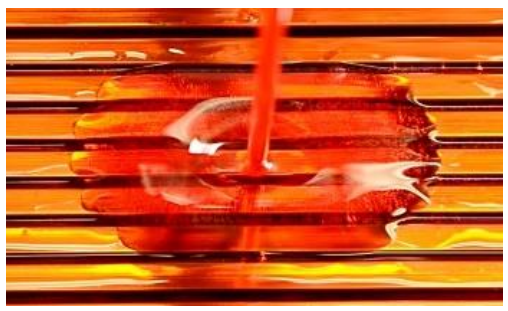

Fig. 4. Experimental result of the oil spreading on the simplified model.

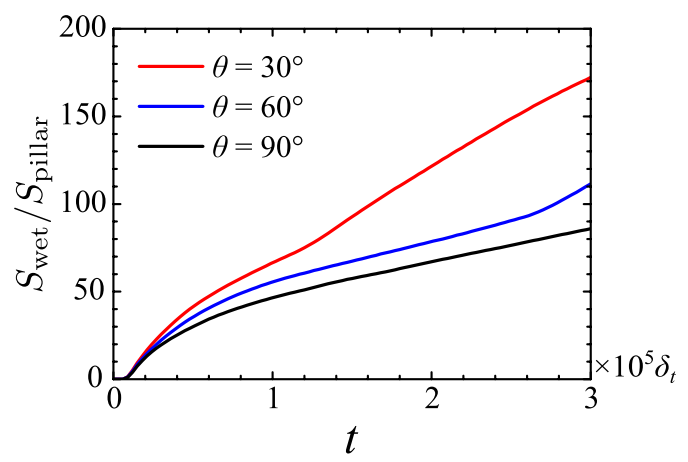

Fig. 5. Transition of the wetting area for each contact angle. 


\subsubsection{Effect of the gaps between the pillars}

In this section, the simulation is performed by changing the gaps of the pillars to $2 \mathrm{~mm}$ and the contact angle to $\theta=30^{\circ}$ from the simulation of the oil in the first section. Figure 6 shows the snapshots of the oil falling on the simplified model with the gaps of $2 \mathrm{~mm}$ between the pillars. It is confirmed that the most oil falls from the pillars and does not step over the gaps. Figure 7 shows the transition of the wetting area for each gap. It is confirmed that the wetting area of the pillars whose gaps are $2 \mathrm{~mm}$ achieves a steady state at an early stage, and there is a large difference in the wetting area compared with the pillars whose gaps are $1 \mathrm{~mm}$.
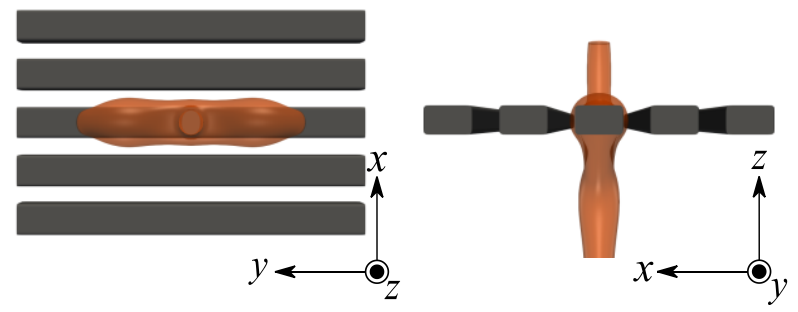

Fig. 6. Snapshots of the oil falling on the simplified model with the gaps of $2 \mathrm{~mm}$ between the pillars.

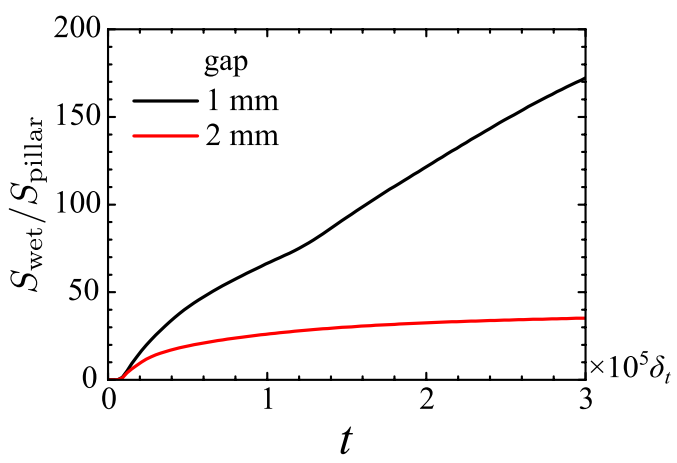

Fig. 7. Transition of the wetting area for each gap.

\subsection{Multi-layered model}

The real-use of the stator has layered coil arrays which is presumed in the simulation as shown in Fig. 8. In this model, four single-layered models are stacked, and the second and fourth layers are rotated $45^{\circ}$ around the central axis normal to the layers. The size of the crosssection of each pillar is $(x, z)=(5 \mathrm{~mm}, 3 \mathrm{~mm})$ and its corners are rounded with a radius of $0.3 \mathrm{~mm}$. The gaps among the pillars are set to $1 \mathrm{~mm}$. In this simulation, the contact angle is set to $\theta=30^{\circ}$. Figure 9 shows the snapshots of the oil falling on the multi-layered simplified model. From this figure, it is confirmed that the oil infiltrates deeply into the pillars. Figure 10 shows the transition of the wetting area of each layer. It is confirmed that the oil infiltrates into the lower layer as time proceeds. Moreover, the wetting area of the second layer eventually becomes larger than that of the first layer. This is because, as shown in Fig. 11, the capillary force acts on both the bottom of the first layer and the top of the second layer in the gap between the first and second layers, while the wetting force acts only on the top of the first layer on the exposed layers.

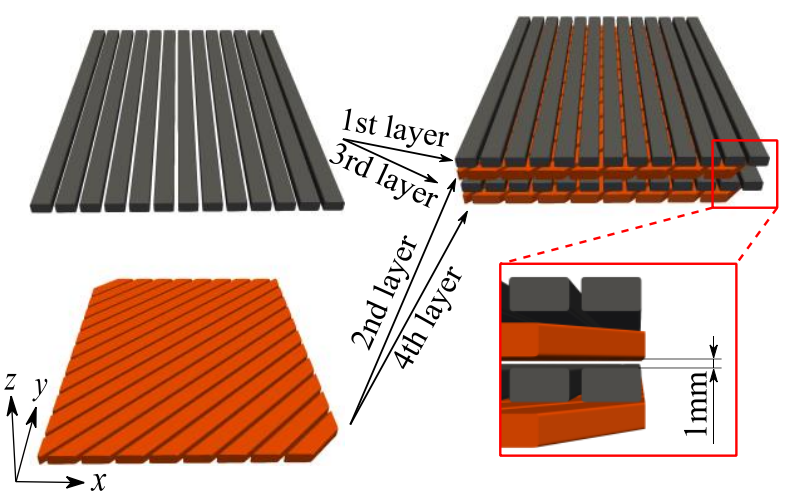

Fig. 8. Schematic drawing of the multi-layered simplified model.
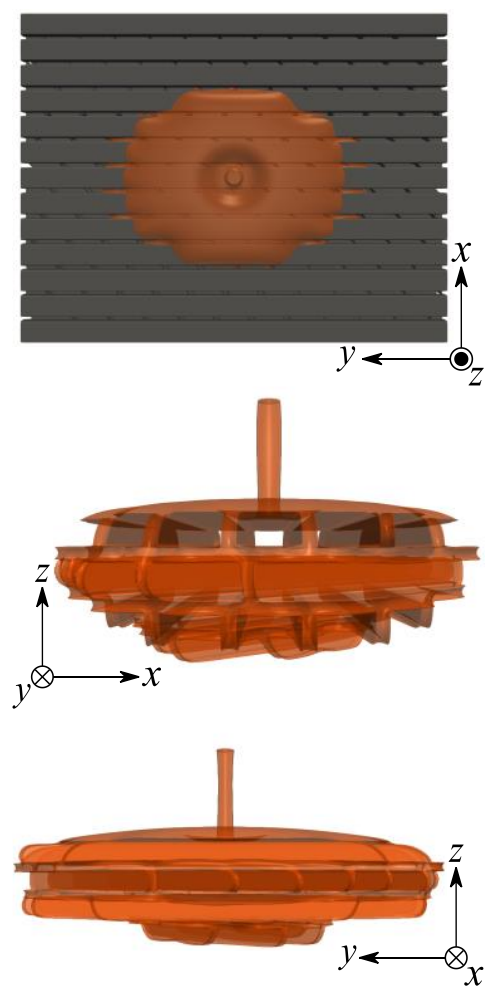

Fig. 9. Snapshots of the oil falling on the multi-layered simplified model.

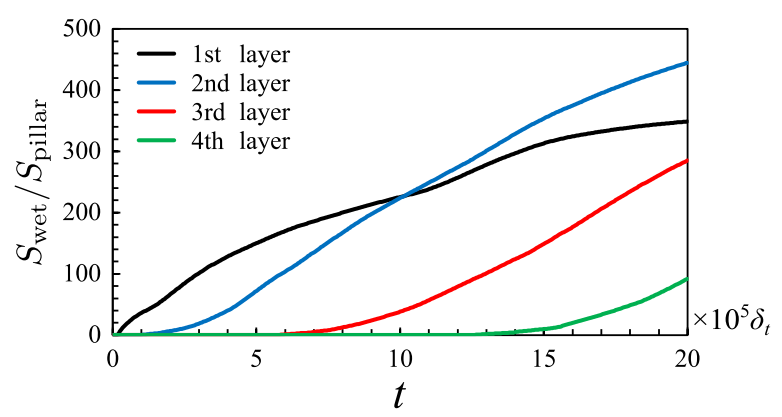

Fig. 10. Transition of the wetting area of each layer. 


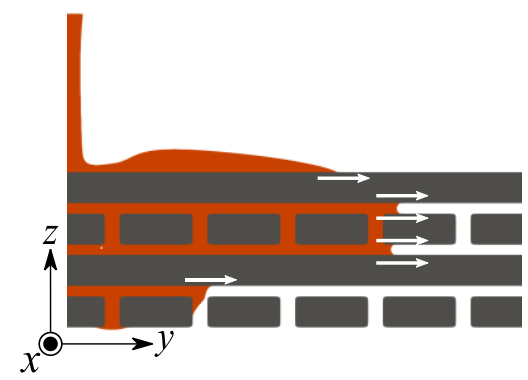

Fig. 11. Schematic drawing of the wetting force acts on the pillar surfaces.

\section{Conclusions}

The liquids falling on the simplified model of the coils are simulated by the lattice Boltzmann method and the phase-field model, and the effects of the governing parameters on the wetting area. It is confirmed that, because of its high viscosity, the oil tends to spread across the pillars. Moreover, the liquid spreads quickly when the contact angle is small. In the case of the multilayered simplified model, the wetting area of the inner pillars is larger than that of the exposed pillars.

This work was supported by "Project for Building Simulation Platforms to Accelerate Development of Next-Generation Vehicles" (TRAMI-Transmission Research Association for Mobility Innovation) grant of 2020.

\section{References}

1. H. Liang, J. Xu, J. Chen, H. Wang, Z. Chai, B. Shi, Phys. Rev. E, 97, 033309 (2018)

2. M. Sugimoto, M. Kaneda, K. Suga, Transactions of the JSME (in Japanese), 86, 20-00014 (2020)

3. J. W. Cahn, J. E. Hilliard, J. Chem. Phys., 28, 258 (1958)

4. P. Bhatnagar, E. Gross, M. Krook, Phys. Rev., 94, $511(1954)$

5. A. Fakhari, D. Bolster, L.-S. Luo, J. Comput. Phys., 341, 22 (2017)

6. H. Liang, B. C. Shi, Z. L. Guo, Z. H. Chai, Phys. Rev. E, 89, 053320 (2014)

7. H. Wang, X. Yuan, H. Liang, Z. Chai, B. Shi, Capillarity, 2, 33 (2019)

8. K. Suga, Y. Kuwata, K. Takashima, R. Chikasue, Comput. Math. Appl., 69, 518 (2015) 